\title{
Identifying Users by Network Traffic Metadata
}

\author{
Gaseb, Alotibi ${ }^{1}$; Nathan, Clarke ${ }^{1,2}$; Fudong, Li $^{1}$ and Steven Furnell ${ }^{1,2,3}$ \\ ${ }^{1}$ Centre for Security, Communications and Network Research (CSCAN) \\ Plymouth University, Plymouth, United Kingdom \\ ${ }^{2}$ Security Research Institute, Edith Cowan University, Western Australia \\ ${ }^{3}$ Center for Research in Information and Cyber Security, \\ Nelson Mandela Metropolitan University, Port Elizabeth, South Africa
}

\begin{abstract}
Insider misuse is become a major threat to many organisations. This is due to the knowledge that might have about the organization's security infrastructure. Therefore, a wide range of technologies have been developed to detect/prevent the insider misuse. Beyond detecting, there is a need to investigate the misuse and identify the individual perpetrating the crime. From a networking perspective, the investigations currently rely upon analysing traffic based upon two approaches: packet-based-approach and flow-based approach. However, a serious limitation in these approaches is the use of IPs addresses to link the misuse to the individual. However, IPs addresses are often not reliable because of the mobile-nature of use (i.e. mobile devices are continually connecting and disconnecting to networks resulting in a device being given a multitude of different IP addresses over time). The presence of DCHP only serves to complicate this for wired environments. This makes it challenging to identify the individual or individuals responsible for the misuse. This paper aims to propose a novel approach that is able to identify using encrypted network traffic. A novel feature extraction process is proposed, that is based upon deriving user actions from network-based applications using packet metadata only. This information is subsequently used to develop biometric-based behavioural profiles. An experiment using 27 participants and 2 months worth of network data is undertaken and shows that users are identifiable with individual applications resulting in recognitions rates of up to $100 \%$.
\end{abstract}

\section{Introduction}

More than 3 billion Internet users have utilized Internet services across the world; and this number is growing continuously [1]. Their daily usage includes (but not limited to) web browsing, entertainment (e.g. watching online videos), communication (e.g. making VoIP calls), finance (e.g. online shopping) and office applications (e.g. Google docs). Indeed, many of the traditional desktop applications such as Office are now found as Internet-based services [2], [3]. According to the Office for National Statistics in the UK (2015), 79\% of the UK population has daily access to the Internet [4]. Moreover, the study has found that smartphone and portable computer devices have a high Internet penetration in the UK [4]. In addition, a significant trend exists in the use of mobile application has been experienced [5] According to the Statistical Portal (2016) the number of mobile applications downloaded has increased on average of $140 \%$ per year between 2012 and 2015 [6].

Malicious attackers can utilize a variety of approaches, such as hacking, Denial of Service (DoS), social engineering and malicious software to launch different attacks [7]. Various studies have shown that they are often successful. Right from an individual to an organisation, anyone can be a victim of security attacks. These attacks are on rise in the recent years. According to a report of McAfee 2014, the estimated annual cost of the cyber-crime to the global economy is more than $\$ 400$ billion, including companies and individuals [8].

Another recent study by Verizone has found that 70 organisations around the world were the victims of information security attacks in 2015 [9]. These studies reflect the extent of security threats being faced by various organizations and the individuals; and they also highlight the importance and the need for effective security systems needed for safeguarding the information systems. In assessing these security threats, it is very essential to understand the various types of threats and the efficiency of the solutions available for mitigating or overcoming them.

These threats can be analysed based on their occurrence or initialization, which can be categorised in to internal and external security threats. Whilst external threats remain an issue, security controls exist to protect outsiders from getting into systems. They do not however often help with the problem of insider threats. Insider threats have grown to be an increasingly significant problem; this is due to the privilege in which the insider user has comparing with the external attacker.

According to Information Security Breaches survey in 2015, insider misuse (e.g. unauthorised access) represents $65 \%$ of the security breaches in the large organisations [10]. In addition, newer technologies such as smartphone and tablets have become one of the main driving factors behind the security breaches. In large organisations, smartphone and tablets have caused $15 \%$ of security or data 
breaches, doubling the percentage comparing with the previous year [10].

Therefore, there is an essential demand for a further investigation to be done behind misuse detection step to identify the individual who committed the crime. Based on networking aspect, the existing insider misuse solutions have relied upon two approaches for identifying insider attacks: packet inspection and flow based approach. They both are used to analyse the network traffic to detect and prevent an attack and have been implemented in different security tools such as Intrusion Detection Systems (IDS), network forensic tools, Security Incident and Event Management (SIEM) systems [11-13]. Whilst these two approaches have their advantages they both fail to truly profile the individual but rather merely the IP address. With the widespread use of mobile technologies (those IP address is constantly changing every time the device is switched on) through to DHCP, where the IP address of a computer can change every time when it is restarted, it is an increasingly unreliable means of undertaking traffic analysis. Furthermore, with an ever increasing volume of encrypted data, deep packet inspection is becoming less useful [14].

Considering these limitations and with a focus on investigating new approaches to handle such limitations, this paper presents the concept and process of deriving user interactions from raw network traffic through the use of metadata independently to user's IP address. Then creating a user-behavioural-based profile that focussed specifically on the user rather than the machine and evaluate the approach across a range of Internet services is the main theme of this study.

The remainder of the paper is structured in the following manner: section 2 provides the background of existing methods of network traffic analysis for insider threats; section 3 presents the creation of application-level user interactions from network level data. The fourth section introduces the proposed approach, followed by an experiment to validate to what extent these user activities are discriminative. A discussion of the findings and how it could be used in practise is presented in section 7. Finally, the conclusions and future work are given in section 8 .

\section{Background}

The research into network traffic analysis started in the 1990s, with the aim of identifying network related attacks [15-16]. Since then, researchers have begun using this technique to investigate different aspects of the problem, such as network behaviour analysis, traffic prioritization, network optimisation and insider misuse. There are two fundamental approaches that can be used to analyse network traffic and being utilised in the existing tools either in detecting/ preventing insider threats: packet or flow based.

\subsection{Packet Based Network Analysis Approach}

The concept of this approach is to perform a bit by bit comparison with predefined signatures of known threats. If there is a similarity between the investigated packets (especially in the payload part) and the threat signature, an attack can be detected. A number of tools (both open source and proprietary) have been developed, including Cain and Abel, TCPDump, Wireshark, Xplico and Microsoft Network Monitor, assisting network security analysts and forensic examiners with an easy analysis of packet information that enable a better understanding of how the attack was formed [17], [18].

However, many limitations have been identified in this approach. One of these is time consuming in analysing the large volumes of data. Another significant issue is the growing use of encryption (e.g. SSL/TLS) within network communications preventing any analysis of the payload [14]. Subsequently, in order to improve the level of performance and its effectiveness various researchers have proposed different methods to deal with deep packet inspection limitation in order to speed up the process in identifying malicious attacks; but they have limited solutions [12], [19-22].

\subsection{Flow Based Network Analysis Approach}

Flow-based approaches seek grouping IP packets passing through an observation point in the computer network within a certain time interval based upon a connection profile. All packets that belong to a specific flow have a set of shared properties. These properties may exist in the header or in other different parts of the packet or both [23]. The advantage of using flows is the vast reduction in data that needs to be analysed in comparison to the packet-based approach.

The flow record normally consists of various fields such as the time and date stamps, the IP addresses of the communication source and destination, their port numbers, the length of the total payload, and the type of protocols. The flow is normally generated from the raw traffic by using third party applications, such as NetFlow, SFlow, JFlow and IPFIX [23-26]. These applications perform different tasks focusing on flow based analysis such as traffic monitoring, identifying unauthorised network activity and tracing the source of DoS attacks. Typically, this is performed by analysing the current traffic flow and identifying any abnormality based upon the historical traffic profile. Based upon this theory, many methods and tools have been proposed and devised within the flow based network analysis domain [27].

With increasing network bandwidth and the use of encryption, the flow-based approach became 
prominent in the market in the area of investigating network traffic issues. In addition, the analysis in large capacity networks is considerably timely and fast. Subsequently, this approach is more efficient in detecting network scans and intrusions, the spreading of malware, and monitoring general network usage. However, whilst the approach solves issues of data volume and encryption, it does not provide additional information on how the user interacts with service but only a service is utilised by an IP address.

Both packet and flow based approaches fundamentally rely upon the IP address as the unique identifier to tag individual. Although, they may successfully achieve certain level of accuracy based on their user identifying mechanism, development of technologies, as previously highlighted, in highly mobile and DHCP-enabled environments, these approaches may not be effective enough in analysing the network traffic. This limitation forces network forensics investigators to examine and analyse larger volumes of raw traffic to identify and correlate misuse, which is a time consuming and expensive activity. Far more useful would be to ask the system to present all traffic belonging to the suspect (or hacked account) and for the system to present this data generated from all devices the individual must be connected to the network through a device (e.g. desktop, smartphone, tablet, and laptop).

\section{User interactions derived from applications level}

For successfully profiling an individual it is important to capture and understand the individual from human-level interactions rather than the machine-to-machine interactions (e.g. network management protocols such as ICMP). Therefore, this approach is focused upon extracting and deriving user based interactions from the raw network data. Intuitively, as users are interacting with Internetbased applications, it should be possible to measure that interaction at the flow/packet level through an understanding of the connection parameters (such as connection type, duration, number of packets, and packet size). The following section explains how user actions can be identified and extracted from network metadata.

\subsection{Methodology}

The approach is based upon the theory that how a user interacts with Internet-based applications on their computer produces a (relatively) unique network packet signature which can subsequently be used to identify the activity.

In order to test this hypothesis, an investigation was undertaken to determine to what extent these signatures could be developed. In the first instance, ten of the most popular internet-based applications were selected for analysis [28]. These applications are Google, YouTube, Skype, Facebook, Dropbox, Hotmail, Twitter, Wikipedia, eBay and BBC. To ensure the resulting analysis was reliable, three researchers were tasked with the collection and analysis of network traces against a predefined set of network captures against user activities (which themselves were repeated 10 times in order to allow for any variance in the resulting network traffic). In our previous paper, an early analysis of these interactions were published which identified that it was possible to determine user interactions with applications through low level network data [29], [30]. The next sections explain the process in determining these signatures in some applications

\subsection{TCP Protocol Signature}

TCP protocol is one of the main protocols utilised in the computer network. Usually three forms of signatures exist in this protocol. First one is 'one packet signature'. Fig 1 shows the patterns when a recipient is added or when a new email button is clicked while using the Hotmail. When the recipient is added or new email button is clicked by a user, one packet is sent from Hotmail server to the client with size 971 bytes.

\begin{tabular}{|lll|}
\hline 204.79 .197 .210 & TCP & 1434 ITCP segnent of a reasse \\
204.79 .197 .210 & TCP & 1434 ITCP segnent of a reasser \\
204.79 .197 .210 & TLSA1.2 & 1016 . Application bata \\
192.168 .200 .58 & TLSA1.2 & 971 . Lpplication data \\
\hline
\end{tabular}

Figure 1. Add recipient on Hotmail

The second type of signature is 'Multiple Packets signature'. When the user starts typing, while chatting on Facebook, 2 packets are sent from the client to a Facebook server. The total size of two of these packets is 1,502 bytes (i.e. $1434+68$ or $1169+333)$. These packets are sent in less than one millisecond timeframe as shown in Fig2.

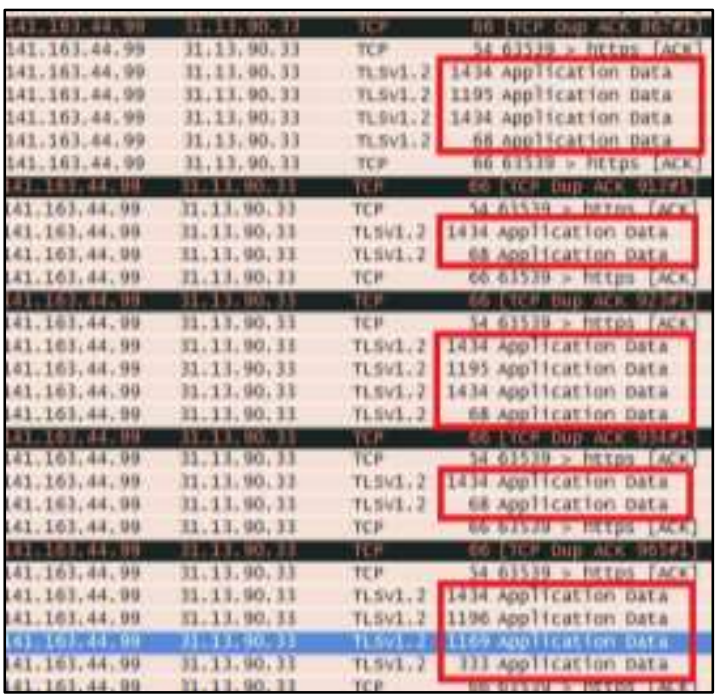

Figure 2 . Typing on Facebook 
The third type is 'stream packets'. Attaching a file in the Hotmail is an example that leads to the initiation of such packets. When the file is attached, stream of packets starts moving from client to Hotmail web server with maximum transmission units (MTU) as shown in Fig3. This process would depend on the file size and would continue till the whole file is attached.

\begin{tabular}{|c|c|c|c|}
\hline LQ.1E. & DH. TAET:ay & $\pi$ & 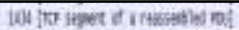 \\
\hline 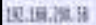 & M-7XY2.29 & $\pi$ & 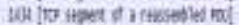 \\
\hline 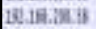 & 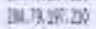 & w & 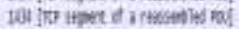 \\
\hline 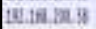 & actasiab & क्र. & 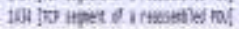 \\
\hline 10.16. & 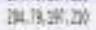 & 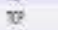 & 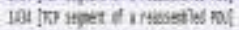 \\
\hline 15:16. & 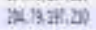 & $x:$ & tai jor unget ef i cumatios oul \\
\hline 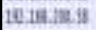 & 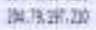 & $x=$ & 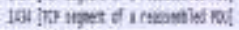 \\
\hline 1e.11 .M.M. & 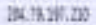 & SSL1 & In woltation int \\
\hline 10.11:.ney & อ. & $w$ & 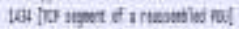 \\
\hline 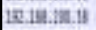 & 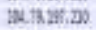 & v & 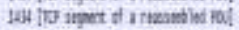 \\
\hline E⿱ & icuara 20 & $w$ & 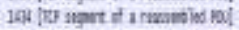 \\
\hline 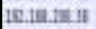 & Whathan & v & 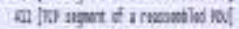 \\
\hline 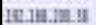 & senuman & v & 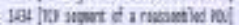 \\
\hline
\end{tabular}

Figure 3. File Attach in Hotmail

\subsection{UDP Protocol Signature}

This type of signature can be observed when a user makes an audio/video call or shares file within the Skype application, a client to client type of connection is established between the both clients. The 'size of packets' is the factor that is used for distinguishing the various activities. The size of the packets is between 1,165 to1,365 bytes during a video call and 129 to147 bytes during an audio call. The activity, 'sending a file' has MTUs and it is between the two Skype users directly as shown in Fig4.

\begin{tabular}{|c|c|c|}
\hline $10 \%$ & 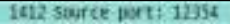 & Destirutian port: solyo \\
\hline tot & 1012 sourch port: 12354 & Destikation port: Se166 \\
\hline Lor & 1212 source port: 12354 & Destination port: $501=6$ \\
\hline (0) & 102 source porti Izas & Destination portl 30150 \\
\hline we: & 12:2 saicce pert: 12354 & Destimation port: satse \\
\hline 100 & 1612 suarce pott: 12394 & Destindition port: soish \\
\hline 109 & 1212 Source port; 12394 & destination port; 90190 \\
\hline 100 & 1212 soirce DoCLI 12354 & Destination Docti Sotse \\
\hline in & 1212 source port1 12354 & osstination port! spos \\
\hline $10 \%$ & 142 sosicce port: 12394 & bestination port: solve \\
\hline 10 & IE12 source port: 1235 s & Destinution portl Sorso \\
\hline ine: & 16t2 source parti 1355 & Dest tration porti sho6 \\
\hline
\end{tabular}

Figure 4 . Sending file in Skype

By applying the same approach upon the 9 services, a set of user interactions identified in each application as demonstrated in Table 1. These patterns are describing the form of user actions when are transmitting through the low network level from client to server or reverse. As can be seen from the table below, there are 9 services from different purposes of usage and from these services 30 user actions have been determined as can be seen in table 1 below. Each user actions signature consists of different parameters such as how many number of packets sent during this action, what was the size of it and what was the direction. According to the table below it seems to be the majority of user action direction has taken a form client/server directions and a few actions that represent in both clients such as some skype application user actions.

Table 1. User actions signatures

\begin{tabular}{|c|c|c|c|c|c|}
\hline \multirow[t]{2}{*}{ Services } & \multirow[t]{2}{*}{ User Action } & \multicolumn{3}{|c|}{ Signature } & \multirow[t]{2}{*}{ Applications IF } \\
\hline & & $\begin{array}{l}\text { No. of } \\
\text { packet }\end{array}$ & $\begin{array}{l}\text { Size of } \\
\text { packet }\end{array}$ & Direction & \\
\hline \multirow{3}{*}{ Hotmail } & File attach. & Stream & MTU & Client>Server & \multirow{3}{*}{$\begin{array}{l}131.253 .61 .2 \\
204.79 .197 .8 \\
204.79 .197 .8\end{array}$} \\
\hline & Compose email & One & 971 & Server-Client & \\
\hline & Insert recipient & One & 971 & Client>Server & \\
\hline \multirow{5}{*}{ Facebook } & Share & Multiple & Various & Server $>$ Client & \multirow{5}{*}{31.13 .8 .8} \\
\hline & Page loading & Stream & MTU & Server>Client & \\
\hline & Attach files & Stream & MTU & Client>Server & \\
\hline & Chat & Multiple & $2,625+$ & Client>Server & \\
\hline & Typing & Multiple & 1,502 & Client>Server & \\
\hline \multirow[b]{3}{*}{$B B C$} & Page Navigation & Stream & Various & Server $>$ Client & \multirow{3}{*}{$\begin{array}{l}212.58 .244 .8 \\
212.58 .246 .8\end{array}$} \\
\hline & Watching Video & Stream & MTU & Server>Client & \\
\hline & Listening Audio & Stream & MTU & Server $>$ Client & \\
\hline \multirow{3}{*}{ Dropbox } & Download files & Stream & MTU & Server $>$ Client & \multirow[b]{3}{*}{108.160 .172 . } \\
\hline & Upload files & Stream & MTU & Client>Server & \\
\hline & Page navigate & Multiple & Various & Serversclient & \\
\hline \multirow{6}{*}{ Skype } & Text messages & One & $794+$ & Client $>$ Server & \multirow{6}{*}{$\begin{array}{l}157.56 .193 . \% \\
157.56 .126 . \%\end{array}$} \\
\hline & Audio calls & Stream & 129.147 & Both Clients & \\
\hline & \begin{tabular}{|l} 
Video calls \\
\end{tabular} & Stream & $1165-1365$ & Both Clients & \\
\hline & File transfer & Stream & MTU & Both Clients & \\
\hline & Click contact & One & 747 & Client>Server & \\
\hline & Idle & One & 587 & Client>Server & \\
\hline \multirow[b]{2}{*}{ Google } & Editing & Multiple & $530+$ & Client $>$ Server & \multirow{2}{*}{$\begin{array}{l}216.58 .208 .8 \\
173.194 .78 .8 \\
216.58 .209 . \text { 충 } \\
\end{array}$} \\
\hline & File download & Stream & MTU & Server $>$ Client & \\
\hline \multirow[b]{2}{*}{ YouTube } & Watch videos & Stream & MTU & Server $>$ Client & \multirow[b]{2}{*}{$216.58 .198 . \%$} \\
\hline & Video upload & Stream & MTU & Client $>$ Server & \\
\hline \multirow{4}{*}{ Twitter } & \begin{tabular}{|l} 
Tweet \\
\end{tabular} & One & $192+$ & Client $>$ Server & \multirow{4}{*}{$\begin{array}{l}185.45 .5 .7 \\
199 \cdot 96.57 . \text { * } \\
104.244 .42 .8\end{array}$} \\
\hline & Upload & Stream & MTU & Client $>$ Server & \\
\hline & Idel/radieng & Multiple & $625+$ & Server $>$ Client & \\
\hline & Click contact & One & 747 & Client $>$ Server & \\
\hline \multirow[b]{2}{*}{ Wikipedia } & Download & Stream & MTU & Server $>$ Client & \multirow[b]{2}{*}{91.198 .174 .8} \\
\hline & Viwing & Stream & MTU & Server $>$ Client & \\
\hline
\end{tabular}


Whilst in our previous paper [30], was found that creating interactions was possible, no further investigation was conducted in measuring these interactions and finding if they could be used to profile individuals at that time. Indeed, the user interaction has consisted of different features that represent the whole user action between the sender and receiver as shown in the Table 2 below. This area of investigation forms and the level of information uniqueness are the fundamental part of this paper

Table 2 . User interactions features

\begin{tabular}{|c|c|c|}
\hline No & Feature Name & Example \\
\hline 1 & Start Time of interaction. & $\begin{array}{c}2014.11 .11 .10: 48 \\
19.769086\end{array}$ \\
\hline 2 & End Time of interaction. & $\begin{array}{c}\text { 2014.11.11.10:48: } \\
19.817979\end{array}$ \\
\hline 3 & Source port number. & 58823 \\
\hline 4 & Service IP. & $216.58 .208 . \%$ \\
\hline 5 & Service port number. & 443 \\
\hline 6 & $\begin{array}{l}\text { \# packets send (client to } \\
\text { server) }\end{array}$ & 2 \\
\hline 7 & $\begin{array}{l}\text { Total size of packets sends } \\
\text { (client to server) }\end{array}$ & 2000 \\
\hline 8 & $\begin{array}{l}\text { \# packets send (server to } \\
\text { client) }\end{array}$ & 6 \\
\hline 9 & $\begin{array}{l}\text { Total size of packets sends } \\
\text { (server to client) }\end{array}$ & 2868 \\
\hline
\end{tabular}

\section{The proposed approach}

The proposed framework consists of two engines: creating Interaction and a biometrics engine. The interaction engine transparently captures the user traffic from the metadata and implement the user action signatures to the related service traffic. This process is created a user interaction as shown in Table 2 for each user across the nine service if the user has traffic in all of them. The biometrics engine is going to check to what extend these user interactions are helpful towards utilising as a fingerprint of the user through the biometric system. Figure 5 illustrates the framework architecture for the user profiling from network traffic via novel application level interactions.

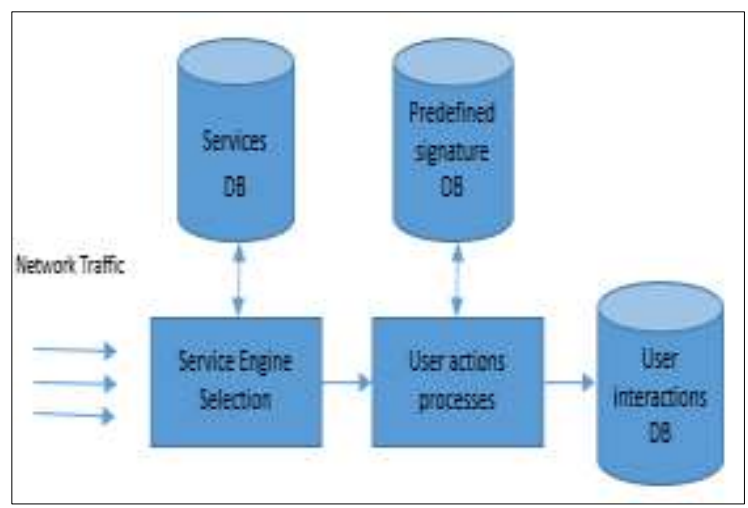

Figure 5. Interactions processes

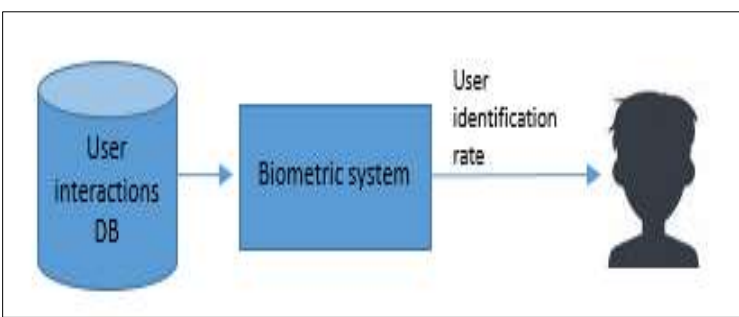

Figure 6. User identification process

The outcomes of interactions processes; which is users' interactions database; is proceed to be an input to the biometrics system as can be seen in figure 6. Finally, the biometric system is going to validate these interactions for each user to gauge the level of uniqueness of this information in order to be utilised as a fingerprint of the user.

\section{Validation of user interactions}

The objective of this experiment is to validate the use of user based interactions as a feature-set and using it within a behavioural profiling biometric system in order to identify the users.

\subsection{Dataset}

The effectiveness and robustness of the experiment is largely dependent upon the quality and quantity of data. As such, a significant data collection activity was undertaken. After a search for openaccess datasets failed to identify any suitable sources, the data was collected centrally from authors research centre. This enabled the researchers to set static IPs within the network in order to provide the ground truth and avoid changing IPs. Twenty-seven participants took part in the collection for 2 months during November 2014 to January 2015. This process was focussed purely on the collection of network metadata. The size of the complete dataset attained at the end was $62.4 \mathrm{~GB}$.

Amongst this traffic, the experiment sought to focus upon nine services that were previously analysed for user interaction signatures; after excluding eBay application due to high level of noisy within identifying signature phase; which include user actions activities in each application from start to finish, source and destination ports, application IP and number and size of packets in each direction as shown in Table 2. Table 3 illustrates a breakdown of the data collected against the chosen nine applications. YouTube, Dropbox, Facebook and Google applications have the largest volume of traffic over than, 21, 17, 5 and 1.8 million packets respectively. After applying the user interaction signatures that were derived from the previous section a vast reduction can be seen as illustrated in the column 3 of Table 3 which represents total number of interactions in each application. This highlights a reduction in data processing in comparison to packet-based 
approaches and also highlights the focus upon interactions rather than flows, which is similar to flow-based approaches. As the data collection was based upon the capture of real data, not all participants were active across all the nine services. However, it is clear that there is sufficient data to test the experimental hypothesis.

Table 3. Applications information

\begin{tabular}{|c|c|c|c|}
\hline App. & No. packets & No. Inter. & $\begin{array}{l}\text { No. } \\
\text { User }\end{array}$ \\
\hline YouTube & $21,131,316$ & $1,322,848$ & 27 \\
\hline Facebook & $5,727,953$ & 386,741 & 27 \\
\hline Google & $1,857,420$ & 194,404 & 27 \\
\hline Twitter & 747,584 & 71,403 & 27 \\
\hline Wikipedia & $1,250,302$ & 5,719 & 20 \\
\hline Hotmail & 703,711 & 122,986 & 19 \\
\hline Dropbox & $17,480,739$ & 98,555 & 16 \\
\hline BBC & 201,263 & 4,180 & 12 \\
\hline Skype & 575,030 & 178,686 & 12 \\
\hline
\end{tabular}

\subsection{Classification Phase}

The classifier has to be applied on the organised data by eliminating the redundant data and converting all input to numeric and this has been done by normalisation phase before the classifier starts [31]. Neural network classifier is considered as an effective approach that deals with complicated patterns. Feed-forward multi-layered perceptron neural network (FF_MLP) was selected for the study because it is useful in complex information processing through using a several layers of adaptive weights which could solve complex non-linear problems [32-33]. The structure of the NN consists of one layer of input (nine inputs), hidden layer with a different values of neurons and one output. Within the FF ML network, supervised learning technique called Levenberg Marquardt (trainlm) was used for training the network and to solve non-linear problem. Neuron sizes have taken the follow values10, 15, 20, 25 and 30 in order to optimise the classifier.

\subsection{Methodology}

The methodology utilised to validate the level of user interactions uniqueness is based upon the standard approach for biometric testing which gives the system an ability to reject imposters and match with the authorised users, which is initiated for all the participants [34]. The experiment is done based on verification model which is repeated for each participant, and in every main participant plays the role of the authorised user and the remaining acts as the impostors. Thus, dividing each user interactions in each application to two halves; one for training the classifier and the other for testing; is a core step because training and testing samples should be separated to ensure that there is no single sample has utilised in both parts. Also, in order to ensure there is sufficient data for both training and testing, a minimum of 30 user interactions is set as a threshold: users will be excluded on an application test if they had less than 30 interactions for that application. The evaluation phase of the classifier performance utilises the True Positive Identification Rate (TPIR) - if the biometric system outputs the identities of the top $t$ matches for each user sample, where $\mathrm{t}$ is representing the rank of accuracy. The following sections are explained each step in details.

\section{Experimental results}

There are a number of key results from our experiment. Figure 7 illustrates the average of TPIR for all users in each application. Applications that have scored a high performance in rank1 considered as a high accurate application in terms of user profile because this reflects the level of discriminative information of user which contribute the classifier towards correct choice. Skype and Hotmail applications have scored high TPIR from rank1 with $98.1 \%$ and $96.2 \%$ TPIR respectively which means the system has correctly classified almost all their samples by assigning them to the correct user. Although, BBC application has got good level of accuracy $81.8 \%$ in rank1, this value has gradually increased to $88.7 \%$, in rank2 and continuous improved up to $95.4 \%$ in rank5. There are some applications scored low TPIR such as YouTube and Dropbox where the system has correctly identified more than $50 \%$ of their samples from the first top value (rank1) but this proportion is continually improved to be roughly $80 \%$ in rank 5 as can be seen in figure 7. Indeed, YouTube application have got the second lowest level of accuracy where its TPIR is $62.8 \%$ in rank1, its interactions represent more than $55 \%$ of whole users' applications as illustrated in Table 3.

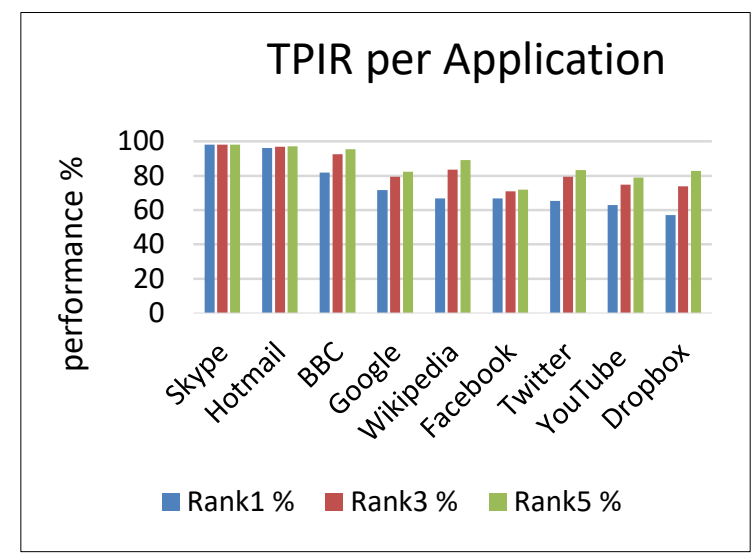

Figure 7. User interactions uniqueness in per application

In terms of individual, the results in table 4 show that concentrating upon the user actions can produce a promising result. According to the table in rank 1, the system has correctly identified about $1 / 2$ of participants with TPIR more than 50\%. Indeed, among these users, there is some users who scored high level of accuracy such as user 9,24 and 27 
where their performance is $68.8 \%, 79.5 \%$ and $88.5 \%$ respectively as can be seen in table 5 . In spite that fact that $50 \%$ of participants has scored promising result as mentioned above, this proportion is improved in rank 5 to being $70 \%$ of users where the system can able to identify their traffic successfully with accuracy $65 \%$ and more. This change of performance is altered from user to another. While some users have scored a sharply increased in their performance between rank 1 and 5 such as user 7 and 22 with almost 50\%, other user scored slightly improvement likewise user 27 as shown in table 4

Table 3. User identification rate

\begin{tabular}{|c|c|c|c|}
\hline $\begin{array}{l}\text { User } \\
\text { ID }\end{array}$ & $\begin{array}{l}\text { TPIR } \\
\text { rank 1 \% }\end{array}$ & $\begin{array}{l}\text { TPIR } \\
\text { rank 3 \% }\end{array}$ & $\begin{array}{l}\text { TPIR } \\
\text { rank 5 \% }\end{array}$ \\
\hline $\mathbf{1}$ & 49 & 55.3 & 63.9 \\
\hline $\mathbf{2}$ & 48.2 & 70.1 & 74.4 \\
\hline $\mathbf{3}$ & 46.3 & 64.8 & 74.4 \\
\hline $\mathbf{4}$ & 65.8 & 78.3 & 82.1 \\
\hline $\mathbf{5}$ & 32.3 & 38 & 51.7 \\
\hline $\mathbf{6}$ & 55.1 & 71.8 & 79.8 \\
\hline $\mathbf{7}$ & 36.9 & 69.1 & 80.2 \\
\hline $\mathbf{8}$ & 60.8 & 67.6 & 68.7 \\
\hline $\mathbf{9}$ & 68.6 & 75.4 & 82.6 \\
\hline $\mathbf{1 0}$ & 39.4 & 56.9 & 63.9 \\
\hline $\mathbf{1 1}$ & 51.2 & 55.1 & 57.9 \\
\hline $\mathbf{1 2}$ & 65.3 & 75 & 78.8 \\
\hline $\mathbf{1 3}$ & 54.1 & 63.3 & 69.5 \\
\hline $\mathbf{1 4}$ & 34.9 & 62.5 & 72.7 \\
\hline $\mathbf{1 5}$ & 59.8 & 80.1 & 84.9 \\
\hline $\mathbf{1 6}$ & 31.1 & 53.2 & 60.1 \\
\hline $\mathbf{1 7}$ & 28.4 & 39.1 & 43.6 \\
\hline $\mathbf{1 8}$ & 64.1 & 73.6 & 75.7 \\
\hline $\mathbf{1 9}$ & 45.5 & 60.1 & 71.2 \\
\hline $\mathbf{2 0}$ & 44.7 & 51.5 & 64.2 \\
\hline $\mathbf{2 1}$ & 50.6 & 71.8 & 87 \\
\hline $\mathbf{2 2}$ & 19.1 & 66.4 & 68.6 \\
\hline $\mathbf{2 3}$ & 41 & 54.4 & 61.7 \\
\hline $\mathbf{2 4}$ & 79.5 & 84.2 & 85.8 \\
\hline $\mathbf{2 5}$ & 53.7 & 61.2 & 68.3 \\
\hline $\mathbf{2 6}$ & 50.2 & 52.9 & 54.8 \\
\hline $\mathbf{2 7}$ & 88.5 & 91.6 & 92.8 \\
\hline & & & \\
\hline
\end{tabular}

Nevertheless, the performance of user profiling of some participants were low as demonstrated in table 4 above, but by exploring the top applications performance of the same users the results were positive. Indeed, all participants have experience at least three applications. Consequently, there are a numbers of promising results have scored across all applications. Table 5 shows the top three applications that scored high level of accuracy from rank1 value per each user. The results reveal that $1 / 3$ of participants have been correctly identified via their interactions with level of accuracy more than $80 \%$ in all top three applications. It is also shown that more than $75 \%$ of the users have got at least one application with $80 \%$ TPIR. While the system has an ability to profile $92 \%$ of the users from their interactions with TPIR more than $74 \%$, there is a small proportion where the system can only assign less than $60 \%$ of the interactions to the correct participant.

Table 4. Users TPIR in rank1 of top three applications

\begin{tabular}{|c|c|c|c|}
\hline \multirow{2}{*}{$\begin{array}{l}\text { User } \\
\text { ID }\end{array}$} & $\mathbf{1}^{\text {st }}$ App. & $\mathbf{2}^{\text {nd }}$ App. & $3^{\text {rd }}$ App. \\
\cline { 2 - 4 } & TPIR\% & TPIR\% & TPIR\% \\
\hline $\mathbf{1}$ & 100 & 95.2 & 50 \\
\hline $\mathbf{2}$ & 94.1 & 74.6 & 74.1 \\
\hline $\mathbf{3}$ & 100 & 60.8 & 59.1 \\
\hline $\mathbf{4}$ & 91.8 & 91.6 & 90.9 \\
\hline $\mathbf{5}$ & 74.1 & 73.6 & 13 \\
\hline $\mathbf{6}$ & 100 & 89.1 & 85.5 \\
\hline $\mathbf{7}$ & 79.2 & 64.8 & 50 \\
\hline $\mathbf{8}$ & 100 & 79.5 & 56.5 \\
\hline $\mathbf{9}$ & 100 & 95 & 93 \\
\hline $\mathbf{1 0}$ & 83 & 63.7 & 56.1 \\
\hline $\mathbf{1 1}$ & 80.5 & 80.3 & 72.7 \\
\hline $\mathbf{1 2}$ & 99.7 & 95 & 80.2 \\
\hline $\mathbf{1 3}$ & 80.9 & 75.3 & 72.7 \\
\hline $\mathbf{1 4}$ & 100 & 62.3 & 48.2 \\
\hline $\mathbf{1 5}$ & 74.5 & 71.1 & 70.9 \\
\hline $\mathbf{1 6}$ & 95.6 & 35.4 & 28 \\
\hline $\mathbf{1 7}$ & 59 & 52 & 30.6 \\
\hline $\mathbf{1 8}$ & 98 & 82 & 67.2 \\
\hline $\mathbf{1 9}$ & 99.4 & 95 & 61.7 \\
\hline $\mathbf{2 0}$ & 75.2 & 73.9 & 63.7 \\
\hline $\mathbf{2 1}$ & 100 & 85.3 & 79.3 \\
\hline $\mathbf{2 2}$ & 43 & 29.4 & 4.2 \\
\hline $\mathbf{2 3}$ & 75.5 & 71.4 & 58.6 \\
\hline $\mathbf{2 4}$ & 100 & 100 & 91.8 \\
\hline $\mathbf{2 5}$ & 80.8 & 75.9 & 51.1 \\
\hline $\mathbf{2 6}$ & 76.1 & 64.8 & 62.4 \\
\hline $\mathbf{2 7}$ & 100 & 100 & 100 \\
\hline & & & \\
\hline & & & \\
\hline
\end{tabular}

Furthermore, the average of TPIR in first application for all users has scored $87.4 \%$, this result has proved that it is possible to use user interaction for creating user profile which is the main aim of this work. Therefore, there is some participants who scored high level of TPIR across the three applications from rank1as shown in (Table 5) such as users 4, 9, 24 and 27, but user 27 has got the best user profile because of the highest level of accuracy got it in all top three applications when the system was able to completely identified all his interactions with $100 \%$ accuracy.

\section{Discussion}

From section 6 it can be seen that user profiling from novel applications interactions is strongly possible. The experiment also reveals that the natural of the user interaction derived from application level is unique, thereby using it to build a user behavioural profile is a promising solution to identify the insider misuse. Moreover, the experiment evidently shown that some applications 
have got a wide unique of user actions such as Skype and Hotmail applications. Accordingly, some users were scored an excellent performance in these applications where the system was able to identify most of their traffic with $100 \%$ level of accuracy as shown in Table 5. The meaning of TPIR being high for some users in various applications is attributed to the level of uniqueness information of a user in this application which makes the discriminative process more easily. Ultimately, using the top three applications based on performance aspect lead to produce an accurate user profile as demonstrated in Table 5.

This provides forensic investigators with a strong approach to identify relevant traffic. When combined with the IP address and windowing (an approach that uses the successful authenticated user interaction to identify the IP address and then uses a windows $+/$ - period (1-2 minutes) to tag all traffic from that IP) provides a very successful approach to target upon the traffic that is most relevant. The use of the IP address in this context is viable as the assumption will only help for a short period of time rather than for the complete duration of the network capture.

This approach of using both the biometric and the IP address suggests that it is not necessary to correctly classify every user interaction but it is important to have at least one application with enough discriminative information for the range of users. Indeed, this approach has providing some positive insights in terms of creating user-behavioral profile from metadata while the system successfully identifying some individual with $100 \%$ of accuracy. Subsequently, reducing the numbers of network traffic analyzing based on user behavioral profile from application level interactions by using a metadata without relying on IP address is a good objective in terms of millions of records needs to be investigated. Therefore, merely sufficient along the timeline for the IP address windows to overlap and provide a confirmation of the IP address.

\section{Conclusions and future work}

The proposed approach is novel, identifying a user from network traffic by using user interactions derives from applications level that give the investigator an ability to overcome upon a serious limitation that exist in the available tools by linked the actions to the belong user regardless the IPs address of the users.

Further work is essential where some users scored low performance and some services do not have enough user actions signatures or lack of usage. Furthermore, to improve the performance different technique need to be implemented in order to have a better performance.

\section{References}

[1] Internetlivestats, "Internet live stats", [online], http://www.internetlivestats.com/internet-users/, date accesed 2nd March 2016.

[2] Microsoft, "Office 365 for business FAQ,[online], https://products.office.com/en-us/business/microsoftoffice-365-frequently-asked-questions, date accessed 1st March 2016.

[3] Library, "Introduction to Google Docs", [online], http://www.lfpl.org/jobshop/docs/google-docs.pdf, date accessed 1st march 2016.

[4] National Statistical in the UK, [online] http://www.ons.gov.uk/peoplepopulationandcommunity/h ouseholdcharacteristics/homeinternetandsocialmediausage /bulletins/internetaccesshouseholdsandindividuals/201508-06, date accessed 15th March 2016

[5] Ofcom (2015): The UK is now a smartphone society,[online], http://media.ofcom.org.uk/news/2015/ cmr-uk-2015/, date accessed 20th March 2016.

[6]Statistical portal,2016, "Application downloaded", [online], http://www.statista.com/statistics/241587/number-of-free-mobile-app-downloads-worldide/, date accessed 25th March 2016.

[7] Microsoft 2016, "Security Threats", [online] https://msdn.microsoft.com/en-us/library/cc723507.aspx, accessed on 14th April 2016.

[8]Mcafee, "Net Losses:Estimating the Global cost of cybercrime", [online] http://www.mcafee.com/us/ resources/reports/rp-economic-impact-cybercrime2.pdf, date accessed: 5 March 2016.

[9]Verizon, "2014 Data Breach Investigations Report", [online], http://www.verizonenterprise.com/DBIR/2014/, date access 28th March 2016

[10] PwC, "2015 Information security breaches survey", [online],https://www.pwc.co.uk/assets/pdf/2015-isbstechnical-report-blue-03.pdf, date accessed: 26th March 2016.

[11] L.D. Merkle, "Automated Network Forensics", Proceedings of the conference on genetic and evolutionary computation (GECCO 2008), pp 1929-1932, in press.

[12] K. Wang and S. J. Stolfo, "Anomalous PayloadBased Network Intrusion Detection", Proceeding of the 7th International Symposium RAID 2004, p.p. 203-222, Sophia Antipolis, France, September 15 - 17, 2004, in press.

[13] QOSMOS (2015) Security information and Event managemnt (SIEM) use case, [online on] http://www.qosmos.com/wpcontent/uploads/2015/08/Qos mos_SIEM_Use-Case_2015.pdf, date accessed 26th March $201 \overline{6}$.

[14] Cisco, "Cisco Visual Networking Index: Forecast and Methodology, 2013-2018", [online],http://www.cisco. $\mathrm{com} / \mathrm{c} / \mathrm{en} / \mathrm{us} / \mathrm{solutions} / \mathrm{collateral} / \mathrm{service}$-provider/ip-ngnip-next-generation-network/white_paper_c11-81360.html, date accessed: 20 December 2014.

[15] G. G. Baehr, W. Danielson, T. L. Lyon, G. Mulligan, M. Patterson, G. C. Scott and C. Turbyfill, "System for packet filtering of data packet at a computer network interface", patent: US5884025, 1995.

[16] K.C. Claffy, H.W. Braun and G.C. Polyzos, "A parameterizable methodology for Internet traffic flow profiling", Selected Areas in Communications, IEEE Journal on , vol.13, no.8, pp.1481-1494, Oct 1995. 
[17] Awodele, O., Oluwabukola, O., Ogbonna, A. C., \& Adebowale, A., "Packet Sniffer - A comparative characteristic evaluation study". Proceedings of Informing Science \& IT Education Conference (InSITE) 2015, 91100

[18] INFOSEC (2016): Password Cracking Using Cain \& Abel, [online],http://resources.infosecinstitute.com/ password-cracking-using-cain-abel/, date accessed 20th March 2016.

[19] I. Ahmed and K. Lhee, "Classification of packet contents for malware detection", Journal in Computer Virology, NOVEMBER 2011, VOLUME 7, ISSUE 4, PP 279-295.

[20] S. Dharmapurikar, P. Krishnamurthy, T. Sproull and J. Lockwood, "Deep packet inspection using parallel Bloom filters", Proceedings of 11th Symposium on High Performance Interconnects, pp.44,51, 20-22 Aug. 2003, in press.

[21] F. Yu, Z. Chen, Y. Diao, T. V. Lakshman and R. H Katz, "Fast and memory-efficient regular expression matching for deep packet inspection", ancs '06 proceedings of the $2006 \mathrm{acm} /$ ieee symposium on architecture for networking and communications systems, pp 93-102, in press.

[22] Y. H. Cho, S. Navab and W. H. Mangione-Smith, "specialized hardware for deep network packet filtering", proceeding of the 12th international conference, pp 452461 , montpellier, france, september $2-4,2002$, in press

[23] B. Claise, "Specification of the IP Flow Information Export (IPFIX) Protocol for the Exchange of IP Traffic Flow Information", IETF, RCF5101, January 2008, [Online], https://tools.ietf.org/html/rfc5101, date accessed: 17 January 2015

[24] Cisco "NetFlow version 9", [online], http://www.cisco.com/c/en/us/products/ios-nx-ossoftware/netflow-version-9/index.html, date accessed 25 February 2015.

[25] Sflow, "sflow", [online] www.sflow.org, date accessed 24 February 2015

[26] Juniper, "Juniper flow monitoring", [online] http://www.juniper.net/us/en/local/pdf/app-

notes/3500204-en.pdf, date accessed 25 February 2015.

[27] B. Li, J. Springer, G. Bebis and M. H. Gunes, "A survey of network flow applications", Journal of Network and Compouter Applications Vol.36 issue 2 pp. 567-581.

[28] eBizMBA(2016): Top 15 Most Popular websites, [online] http://www.ebizmba.com/articles/most-popularwebsites,date accessed 1st September 2016.

[29] Alotibi G, Li F, Clarke NL, Furnell SM (2015): "Behavioral-Based Feature Abstraction from Network Traffic", 10th International Conference on Cyber warfare and Security, Kruger National Park, South Africa, 24-25 March, pp1-9, ISBN 978-1-910309-97-1, 2015.

[30] Li.F, Clarke.N, Alotibi.G \& Joy.D(2015): "Forensic Investigation of Network Traffic: A Study into the Derivation of Application-Level features from NetworkLevel Metadata", Big data, Could and Security (ICTBDCS 2015), 27-28 July, ISSN: 2382-5669, pp68-73, 2015 .

[31] J. Sola and J. Sevilla (1997) "Importance of Input Data Normalization for the Application of Neural Networks to Complex Industrial Problems", IEEE TRANSACTIONS ON NUCLEAR SCIENCE, VOL. 44 NO. 3, JUNE 1997.
[32] Y. Chtioui, D. Bertrand, M. Devaux, D. Barba (1997): Comparison of multilayer perceptron and probabilistic neural networks in artificial vision application to the discrimination of seeds, Journal of Chemometrics 11 (1997) $111-129$.

[33] Amit Mehta, Arjun Singh Parihar and Neeraj Mehta (2015): Supervised Classification of Dermoscopic Images using Optimized Fuzzy Clustering based Multi-Layer Feed-Forward Neural Network, IEEE International Conference on Computer, Communication and Control, Indore, 10-12 Sept. 2015.

[34] Nathan Clarke, Steven Furnell \& Benn Lines (2004) “ Application of Keystrock Analysis to Mobile Text Messageing", Proceedings of the 3rd Security Conference, Las Vegas, USA, 14-15 April, 2004.

[35] Skype, "Which ports need to be open to use Skype for Windows desktop", [online] https://support.skype. com/en/faq/FA148/which-ports-need-to-be-open-to-useskype-for-windows-desktop, date accessed 4th March 2016. 
International Journal of Chaotic Computing (IJCC), Volume 4, Issue 2, December 2016 